\title{
Periodic Disturbance Rejection of a PMSM with Adaptive Control Algorithms
}

\author{
Sebastian Maier \\ Department of System Dynamics \\ and Control \\ Institute of Robotics and Mechatronics \\ German Aerospace Center (DLR) \\ 82234 Wessling, Germany \\ Muenchner Str. 20 \\ Email: Sebastian.Maier@dlr.de
}

\author{
Johann Bals \\ Department of System Dynamics \\ and Control \\ Institute of Robotics and Mechatronics \\ German Aerospace Center (DLR) \\ 82234 Wessling, Germany \\ Muenchner Str. 20 \\ Email: Johann.Bals@dlr.de
}

\author{
Marc Bodson \\ Department of Electrical \\ and Computer Engineering \\ University of Utah \\ 50 S Central Campus Dr. \\ Salt Lake City, UT 84112, USA \\ Email: Bodson@ece.utah.edu
}

\begin{abstract}
Permanent magnet synchronous motors (PMSMs) produce a parasitic oscillating torque due to several reasons. This contribution cancels the oscillating torque with adaptive control algorithms. Therefore a mathematical model of the PMSM is necessary. A model with nonlinear dynamics and a Fourier approach for the ripples is used as a mathematical description. Through comparisons between measured data and simulated data it is shown that the model assumptions are valid.

The adaptive algorithm is implemented as an add-on controller to the already existing control system which consists of a feedforward part and a basis controller. The challenge is that the closed loop system has a resonant frequency and the algorithm should have the same performance for all frequencies. Experimental results show the performance and convergence of the adaptive algorithm at constant and non constant velocity.
\end{abstract}

\section{INTRODUCTION}

In many industrial applications, like robotics or machine tools, permanent magnet synchronous motors play a very important role. A bad property of PMSMs is that they generate torque ripples which cause a robot oscillating at tool center point (TCP). This effect is insignificant if a robot does pick and place tasks, because its structure dynamics is the most important issue. Whenever a robot does quite slow movements the ripples, generated by the motor, play an important role. For practical robot applications like laser welding or gluing the oscillations of the TCP are a disadvantage for the workpiece. A PMSM often comes as servo-motors, where a cascaded control structure is used. The inner loop is the current loop with a decoupling network and the outer loop is a speed or position loop.

Control based torque ripple minimization was studied by many researchers already. The activities split into feedforward and feedback control methods, see the summary of [7] for AC drives. Several feedback control methods were discussed, which implement the internal model principle [4], iterative learning control with an observer [14], adaptive linear neuron estimation [10], disturbance observers [3] or adaptive control [11]. Also same feedforward methods are proposed like in [6]. The main properties of our method is the fast convergence, it can handle accelerated movements and measurement noise. In

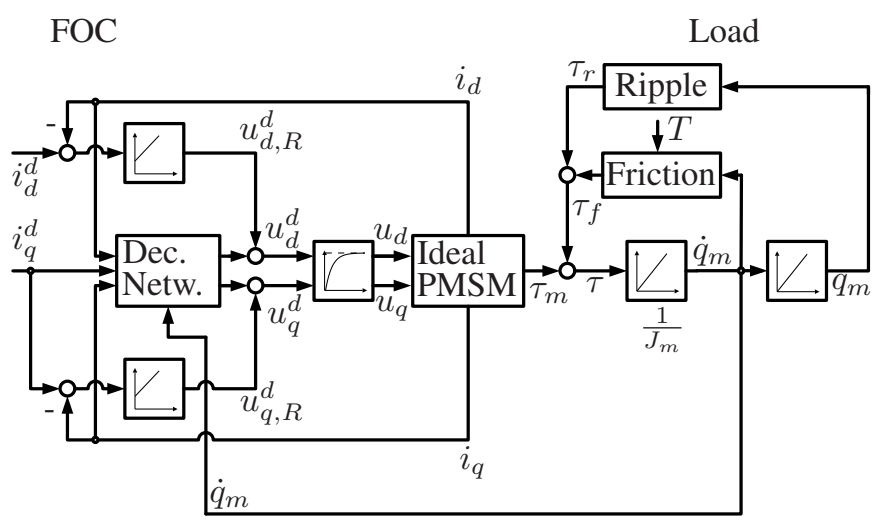

Fig. 1. Block diagramm of a servo PMSM.

steady state the disturbance is canceled totally. Moreover, this method can be combined with any basis feedback controller and with any feedforward controller based on an inverse model.

\section{MODELING OF A PMSM}

Figure 1 shows, how the physics of a PMSM can be described by an analytical mathematical model. The ideal PMSM is described by nonlinear differential equations in the $d q$-coordinate system (CS). To this ideal model friction and torque ripples are added. Often a PMSM is run with a field oriented control (FOC) which consists of linear PI controllers, a decoupling network and an inverter.

\section{A. Nonlinear differential equations in $d q-C S$}

The electrical differential equations of the PMSM are given by

$$
\begin{aligned}
L_{d} \frac{d i_{d}}{d t} & =u_{d}+\frac{d q_{e l}}{d t} L_{q} i_{q}-R i_{d} \\
L_{q} \frac{d i_{q}}{d t} & =u_{q}-\frac{d q_{e l}}{d t} L_{d} i_{d}-R i_{q}-\dot{q}_{e l} \psi_{d m}
\end{aligned}
$$

with inductance $L$, resistance $R$, electric angle $q_{e l}$, current $i$, voltage $u$ and the flux $\psi_{d m}$. The indexes $d$ and $q$ describe the 
axis of the $d q \mathrm{CS}$. The electromagnetic torque is given by

$$
\tau_{m}=\frac{3}{2} n_{p} \psi_{d m} i_{q}+\left(L_{d}-L_{q}\right) i_{d} i_{q}
$$

where $\frac{3}{2} n_{p} \psi_{d m}$ corresponds to the $k_{T}$-factor of a DC-machine. The mechanical differential equation is given by

$$
J_{m} \frac{d^{2} q_{m}}{d t^{2}}=\tau_{m}-\tau_{f}\left(T, \dot{q}_{m}\right)-\tau_{r}\left(q_{m}\right)
$$

with the moment of inertia $J_{m}$, friction torque $\tau_{f}$, ripple torque $\tau_{r}$. For later control methods it is important, that only the current $i_{q}$ and the motor position $q_{m}$ are measurable. The exact derivation of the equations can be found in references [12] and [2].

\section{B. Causes for the torque ripples}

There are several reasons for torque ripples in electric machines, see [15] [7] and [13], where also design modifications are discussed to minimize the torque ripples. Some ripples arise due to harmonics in the field flux linkage which are electromagnetic effects. Another effect is often called cogging, which is an unintentional interaction between the edge of the pole and the slot, which creates a magnetic reluctance. Another reason for ripples are due to current offsets and current scaling errors. Nevertheless, for control based ripple rejection we assume the following.

Assumption 1: The torque ripples can be modeled on torque level independent of their cause with

$$
\tau_{r}=\sum_{N=1}^{\infty} m_{N} \cos \left(N q_{m}+\varphi_{N}\right)
$$

\section{Basis control of a PMSM}

A servo PMSM is often run with FOC, which consists of several parts. In the $d$-path and $q$-path often two PI current controllers

$$
P I_{i_{q}, i_{d}}(s)=k_{s} \frac{T_{s} s+1}{T_{s} s}
$$

are used. The equations for the decoupling network are given by

$$
\begin{aligned}
\Delta u_{d} & =-\dot{q}_{e l} L_{q} i_{q} \\
\Delta u_{q} & =\dot{q}_{e l} L_{d} i_{d}+\dot{q}_{e l} \psi_{d m},
\end{aligned}
$$

the voltages $u_{d, q}$ are the outputs of the linearization. The inverter is often realized by a pulse width modulation (PWM), which is from the control point of view an ideal delay given by

$$
P W M_{d, q}(s)=e^{-T_{\sigma} s},
$$

with the cycle time of the PWM $T_{\sigma}$. Since an ideal delay sometimes causes a problem in simulation or controller design the ideal delay can be approximated as a first order system or Pad Approximation. The outer control loop is a speed controller or a position controller. In our case a position controller is used with a PID structure given by

$$
P I D_{q_{m}}(s)=k_{p}+\frac{k_{i}}{s}+\frac{k_{d} s}{T_{d} s+1} .
$$
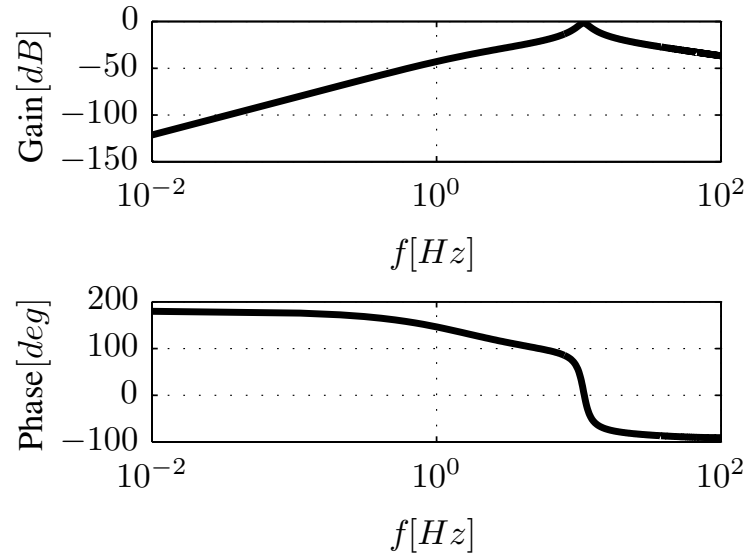

Fig. 2. Bode diagram from torque ripple to motor velocity (disturbance transfer function).

All these transfer functions result in linearized closed loop transfer functions. The effect of the ripples on the motor velocity is analyzed with the closed-loop disturbance transfer function which is shown in figure 2. This figure shows the different effects of the ripples for different frequencies. The frequencies of the Bode diagram are proportional to the velocity of the motor, so at the resonant region around $10 \mathrm{~Hz}$ the ripple have the biggest effect on the system's output (motor position).

\section{Fit of simulation and experiment}

The step response of the motor is shown in figure 3 which is the comparison between simulation and experiment. The figure shows a good correlation in transient and steady state behavior of current $i_{q}$ and speed $q_{m}$. At the transient operation the motor dynamic plays the most important role and in steady state the motor ripple and friction get more important. In this case the $i_{q}$ signal shows an offset, which is achieved with a friction model given by

$$
\tau_{f}=\tau_{f}\left(\dot{q}_{m}(t), T\right)
$$

where $T$ is the motor temperature. The friction model was found at different motor velocities and is then put into a lookup table.

\section{AdAptive Control Schemes}

This paper introduces two different algorithms for periodic disturbance rejection which are called $G^{-1}$ algorithm and filtered least mean squared (FXLMS) algorithm. Since the plant is not strictly positive real (SPR) some system information is needed, so the $G^{-1}$-algorithm uses an inverse system matrix and the FXLMS algorithm uses a model of the plant as a filter, see figure 4.

These type of controllers fall in the framework of adaptive feedforward cancellation (AFC) which was introduced by Bodson [1] and [5] for the suppression of sound waves (active noise control). An important part of this work is to adjust these gradient based algorithms to mechatronic systems. 

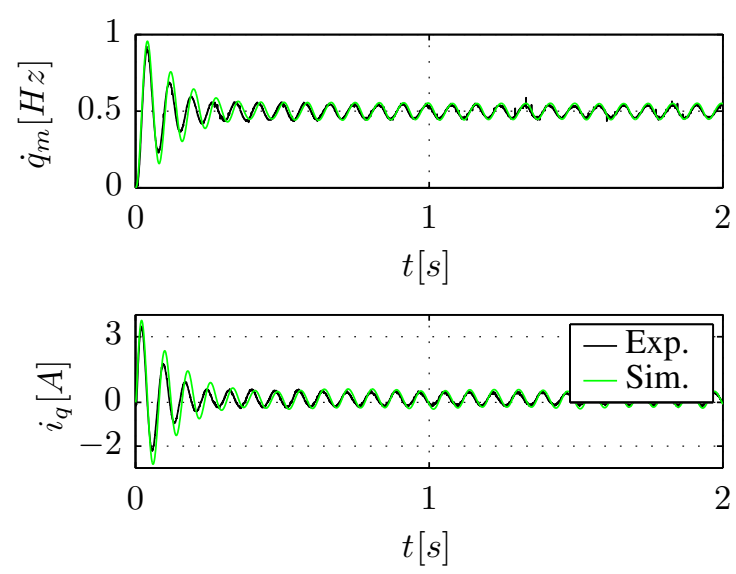

Fig. 3. Comparison of simulation and experiment after a step response on velocity level.

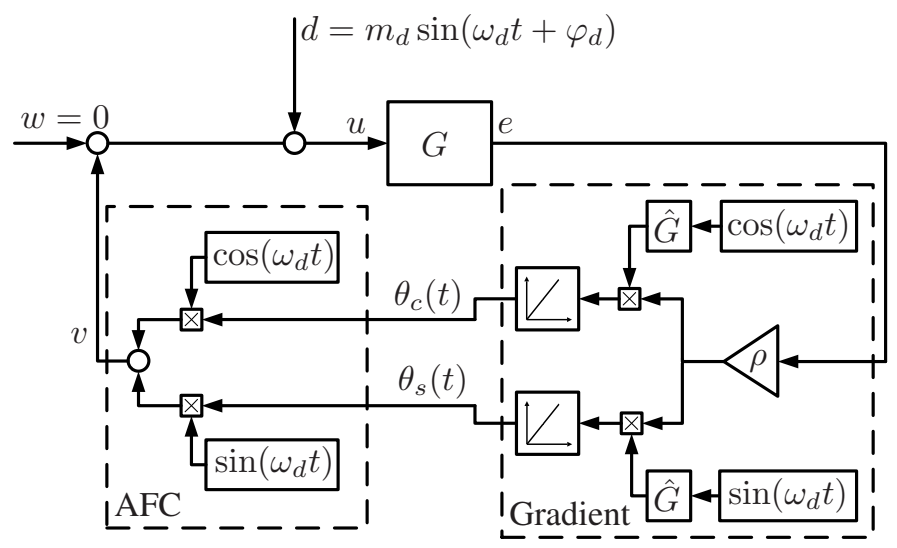

Fig. 4. Block diagram of AFC and gradient.

1) The FXLMS algorithm: The following derivation is only valid for plants without any reference input. The disturbance is given in the time domain and is transformed to vector notation

$$
\begin{aligned}
d(t) & =\theta_{c}^{*} \cos \left(\omega_{d} t\right)+\theta_{s}^{*} \sin \left(\omega_{d} t\right) \\
& =\left[\begin{array}{c}
\cos \left(\omega_{d} t\right) \\
\sin \left(\omega_{d} t\right)
\end{array}\right]^{T}\left[\begin{array}{l}
\theta_{c}^{*} \\
\theta_{s}^{*}
\end{array}\right] \\
& =\boldsymbol{w}^{T}(t) \boldsymbol{\theta}^{*},
\end{aligned}
$$

where $\boldsymbol{w}$ is the regressor or regressor vector. The control signal $v(t)$ is given by

$$
\begin{aligned}
v(t) & =\theta_{c}(t) \cos \left(\omega_{d} t\right)+\theta_{s}(t) \sin \left(\omega_{d} t\right) \\
& =\left[\begin{array}{c}
\cos \left(\omega_{d} t\right) \\
\sin \left(\omega_{d} t\right)
\end{array}\right]^{T}\left[\begin{array}{l}
\theta_{c}(t) \\
\theta_{s}(t)
\end{array}\right] \\
& =\boldsymbol{w}^{T}(t) \boldsymbol{\theta}(t) .
\end{aligned}
$$

Since the reference signal is $w=0$, the output of the plant is given by

$$
\begin{aligned}
e(t) & =G(s)[v(t)-d(t)]^{1} \\
& =G(s)\left[\boldsymbol{w}^{T}(t)\left(\boldsymbol{\theta}(t)-\boldsymbol{\theta}^{*}\right)\right]
\end{aligned}
$$

and is the error signal for the adaptation. Perfect cancellation $(e=0)$ is achieved, if $\boldsymbol{\theta}^{*}=\boldsymbol{\theta}(t)$. This can be obtained by an adaptation such that $\boldsymbol{\theta}(t) \rightarrow \boldsymbol{\theta}^{*}$. A gradient based algorithm is used that minimizes the squared error $e(t)^{2}$ as a function of the adaptive states $\boldsymbol{\theta}(t)$, where $\rho$ is the gain of the adaptation. The equations of the adaption law is given by

$$
\begin{aligned}
\dot{\boldsymbol{\theta}}(t) & =-\rho \nabla e^{2}(t) \\
& =-\rho \frac{\partial e^{2}(t)}{\partial \boldsymbol{\theta}(t)} \\
& =-2 \rho \hat{G}(s)[\boldsymbol{w}(t)] e(t),
\end{aligned}
$$

where $\hat{G}(s)\left[\boldsymbol{w}^{T}(t)\right]$ is the inner derivative of the error. In the continuous time domain the adaptation can be written as

$$
\dot{\boldsymbol{\theta}}(t)=-2 \rho \hat{G}(s)[\boldsymbol{w}(t)] e(t),
$$

which is an implementation of the algorithm. Stability can not be proven $\forall \rho>0$, which is a drawback of the algorithm. Still the following lemma can be proven with Lyapunov theory, see [8].

Lemma 1: With assumption 2 and assumption 3 stability can be proven for the adaptation law $\dot{\boldsymbol{\theta}}(t)=$ $-2 \rho \hat{G}(s)[\boldsymbol{w}(t)] e(t)$ with Lyapunov theory in all operating points.

Assumption 2: The transfer function of the plant $G(s)=$ $\hat{G}(s)$ is completely known.

Assumption 3: The adaptation gain is chosen to be small enough, it holds for the coordinates $\dot{\phi}(t) \cong 0$ and $\phi(t) \cong$ const with the coordinate transformation $\phi(t)=\boldsymbol{\theta}(t)-\boldsymbol{\theta}^{*}$ and $\dot{\phi}(t)=\dot{\boldsymbol{\theta}}(t)$.

Proof 1: If the error signal from equation 14 is inserted into the adaptation law 18 it follows

$$
\dot{\boldsymbol{\theta}}(t)=-2 \rho G(s)[\boldsymbol{w}(t)] G(s)\left[\boldsymbol{w}^{T}(t)\left(\boldsymbol{\theta}(t)-\boldsymbol{\theta}^{*}\right)\right]
$$

and with the coordinate transformation

$$
\dot{\phi}(t)=-2 \rho G(s)[\boldsymbol{w}(t)] G(s)\left[\boldsymbol{w}^{T}(t) \boldsymbol{\phi}\right] .
$$

With assumption 3 it follows

$$
\dot{\boldsymbol{\phi}}(t)=-2 \rho G(s)[\boldsymbol{w}(t)] G(s)\left[\boldsymbol{w}^{T}(t)\right] \boldsymbol{\phi}(t) .
$$

To simplify the notation it holds $G(s)[\boldsymbol{w}(t)]=\boldsymbol{w}_{F}(t)$ which leads to the nonlinear differential equation

$$
\begin{aligned}
\dot{\boldsymbol{\phi}}(t) & =-2 \rho \boldsymbol{w}_{F}(t) \boldsymbol{w}_{F}^{T}(t) \boldsymbol{\phi}(t), \\
& =-2 \rho \boldsymbol{A}^{*}(t) \boldsymbol{\phi}(t)=-\boldsymbol{A}(t) \boldsymbol{\phi}(t) .
\end{aligned}
$$

As an approach for the Lyapunov function

$$
V(t)=\phi^{T}(t) \phi(t)
$$

is chosen. For the derivative of the Lyapunov function it holds

$$
\begin{aligned}
\dot{V}(t) & =\dot{\boldsymbol{\phi}}^{T}(t) \boldsymbol{\phi}(t)+\boldsymbol{\phi}^{T}(t) \dot{\boldsymbol{\phi}}(t), \\
& =-(\boldsymbol{A}(t) \boldsymbol{\phi}(t))^{T} \boldsymbol{\phi}(t)-\boldsymbol{\phi}^{T}(t) \boldsymbol{A}(t) \boldsymbol{\phi}(t), \\
& =-\boldsymbol{\phi}(t)^{T} \boldsymbol{A}(t)^{T} \boldsymbol{\phi}(t)-\boldsymbol{\phi}^{T}(t) \boldsymbol{A}(t) \boldsymbol{\phi}(t), \\
& =-\boldsymbol{\phi}^{T}(t)\left(\boldsymbol{A}(t)+\boldsymbol{A}^{T}(t)\right) \boldsymbol{\phi}(t)
\end{aligned}
$$

\footnotetext{
${ }^{1}$ For notation convolution is written as $G(s)[v(t)-d(t)]=g(t) *(v(t)-$ $d(t))$ in squared brackets, so time domain signals and frequency domain system can mixed up easily.
} 
The condition of stability is fulfilled if $\boldsymbol{A}(t)+\boldsymbol{A}^{T}(t)$ is positive semidefinite, which is the case, because $\boldsymbol{A}(t)+\boldsymbol{A}^{T}(t)$ is symmetric and has the eigenvalues $\lambda=0,2$.

\section{A. The $\boldsymbol{G}^{-1}$ algorithm}

Another implementation of the gradient algorithm is described by the $G^{-1}$ algorithm and the adaptation law is given by

$$
\dot{\boldsymbol{\theta}}(t)=-2 \rho \hat{\boldsymbol{G}}^{-1}(\omega) \boldsymbol{w}(t) e(t)
$$

with the system matrix

$$
\hat{\boldsymbol{G}}(\omega)=\left[\begin{array}{cc}
\Re\{\hat{G}(j \omega)\} & \Im\{j \hat{G}(\omega)\} \\
-\Im\{\hat{G}(j \omega)\} & \Re\{j \hat{G}(\omega)\}
\end{array}\right] .
$$

For the error signal in matrix notation it follows

$$
e(t)=\boldsymbol{w}(t)^{T} \boldsymbol{G}(\omega)\left(\boldsymbol{\theta}(t)-\boldsymbol{\theta}^{*}\right) .
$$

For the proof of stability the following lemma has to be proven

Lemma 2: With assumption 2 stability can be proven for the adaptation low $\dot{\boldsymbol{\theta}}(t)=-2 \rho \hat{\boldsymbol{G}}^{-1}(\omega) \boldsymbol{w}(t) e(t)$ with Lyaponov in all operating points.

Proof 2: If the error signal from equation 31 is inserted into the adaptation law 29 it follows

$$
\dot{\boldsymbol{\theta}}(t)=-2 \rho \boldsymbol{G}^{-1}(\omega) \boldsymbol{w}(t) \boldsymbol{w}(t)^{T} \boldsymbol{G}(\omega)\left(\boldsymbol{\theta}(t)-\boldsymbol{\theta}^{*}\right)
$$

and with the coordinate transformation $\phi(t)=\boldsymbol{\theta}(t)-\boldsymbol{\theta}^{*}$, $\dot{\phi}(t)=\dot{\boldsymbol{\theta}}(t)$ it follows

$$
\begin{aligned}
\dot{\boldsymbol{\phi}}(t) & =-2 \rho \boldsymbol{G}^{-1}(\omega) \boldsymbol{w}(t) \boldsymbol{w}(t)^{T} \boldsymbol{G}(\omega) \boldsymbol{\phi}(t) \\
& =-\boldsymbol{G}^{-1}(\omega) \boldsymbol{A}(t) \boldsymbol{G}(\omega) \boldsymbol{\phi}(t) .
\end{aligned}
$$

As an approach for the Lyapunov function

$$
V(t)=\phi^{T}(t) \phi(t)
$$

is chosen. For the derivative of the Lyapunov function it holds

$$
\begin{aligned}
\dot{V}(t) & =\dot{\boldsymbol{\phi}}^{T}(t) \boldsymbol{\phi}(t)+\boldsymbol{\phi}^{T}(t) \dot{\boldsymbol{\phi}}(t), \\
& =-\left(\boldsymbol{G}^{-1} \boldsymbol{A} \boldsymbol{G} \boldsymbol{\phi}\right)^{T} \boldsymbol{\phi}-\boldsymbol{\phi}^{T} \boldsymbol{G}^{-1} \boldsymbol{A} \boldsymbol{G} \boldsymbol{\phi}, \\
& =-\boldsymbol{\phi}^{T} \boldsymbol{G}^{T} \boldsymbol{A}^{T}\left(\boldsymbol{G}^{-1}\right)^{T} \boldsymbol{\phi}-\boldsymbol{\phi}^{T} \boldsymbol{G}^{-1} \boldsymbol{A} \boldsymbol{G} \boldsymbol{\phi}, \\
& =-\boldsymbol{\phi}^{T}\left(\boldsymbol{G}^{T} \boldsymbol{A}^{T}\left(\boldsymbol{G}^{-1}\right)^{T}+\boldsymbol{G}^{-1} \boldsymbol{A} \boldsymbol{G}\right) \boldsymbol{\phi}
\end{aligned}
$$

The condition of stability is fulfilled if $\boldsymbol{G}^{T} \boldsymbol{A}^{T}\left(\boldsymbol{G}^{-1}\right)^{T}+$ $G^{-1} A G$ is positive semidefinite, which is the case, because $\boldsymbol{G}^{T} \boldsymbol{A}^{T}\left(\boldsymbol{G}^{-1}\right)^{T}+\boldsymbol{G}^{-1} \boldsymbol{A} \boldsymbol{G}$ is symmetric and has the eigenvalues $\lambda=0,2$.

\section{REJECTION OF TORQUE RIPPLES AT A PMSM}

In this paper we use gradient based AFC methods to reject torque ripples of a PMSM. The disturbance can not be modeled as an exosystem, but as a function of a system state (motor position), see equation 5 .

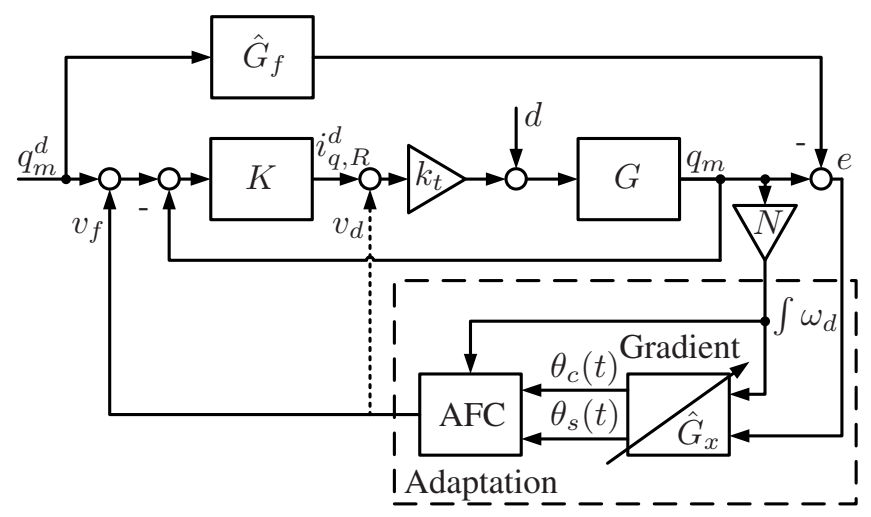

Fig. 5. Block diagram of the complete control system for the PMSM without feedforward control.

\section{A. Integration of the adaptation in the existing control system}

Several signals can be used as an error signal and also several locations for the intervention of the adaptive signal $\left(v_{d}, v_{f}\right)$ are possible, see figure 5 . If the adaptive signal intervenes on the position level the adaptive states are not constant at accelerated movements, because of the dynamics, which is between the location of intervention and the location of the disturbance. If the location of intervention of the adaptive signal is the current level, it is possible to achieve (quasi) constant adaptive states. The only disadvantage of this algorithm is that it uses the closed loop disturbance transfer function of the closed loop system, which is a function of the basis controller. Thus, if the basis control system changes, also the adaption has to be adjusted.

As an error signal the position signal is used because it is less noisy and the velocity has to be calculated numerically. It is also good-natured during accelerated movements.

1) Adaptation without feedforward control: The goal of the control system of figure 5 is to follow a reference trajectory and to reject a periodic disturbance at the same time. It holds for the motor position with location of intervention of the adaptive controller on position level

$$
q_{m}=G_{f} v_{f}+G_{f} q_{m}^{d}+G_{d} d .
$$

with $G_{f}=\frac{G K}{1+G K}$ and $G_{d}=\frac{G}{1+G K}$. For the adaptation we make the system fictively free from the reference trajectory, which is achieved with

$$
e=q_{m}-\hat{G}_{f} q_{m}^{d}
$$

The error for the adaptation becomes with equation 40 and assumption 2

$$
e(t)=G_{d}(s)\left[\boldsymbol{w}^{T} \boldsymbol{\theta}^{*}\right]+G_{f}(s)\left[\boldsymbol{w}^{T} \boldsymbol{\theta}(t)\right] .
$$

The adaptation error is minimized with a gradient so it follows

$$
\begin{aligned}
\dot{\boldsymbol{\theta}} & =-\rho \frac{\partial e^{2}(t)}{\partial \boldsymbol{\theta}(t)} \\
& =-2 \rho G_{f}(s)\left[\boldsymbol{w}^{T} e(t)\right] .
\end{aligned}
$$




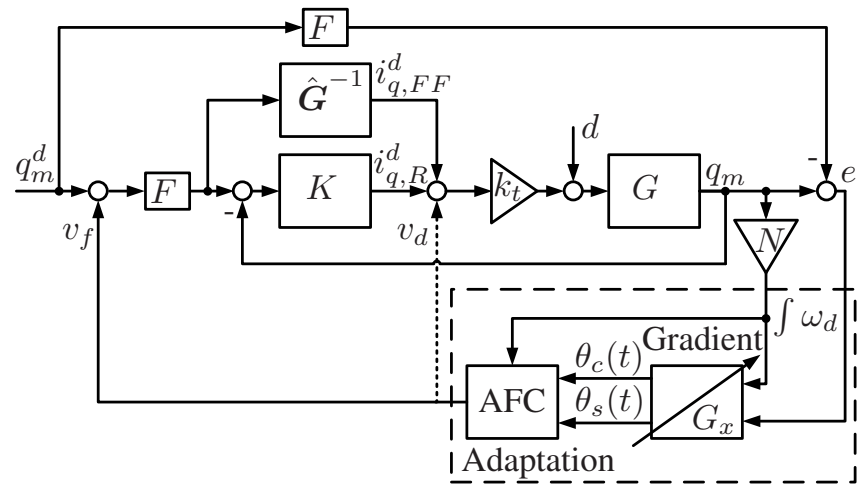

Fig. 6. Block diagram of the complete control system for the PMSM without feedforward control.

The filter of the adaptation of figure $5 \hat{G}_{x}(t)$ is the reference transfer function $\hat{G}_{f}(s)$. If the location of intervention is chosen to be current level it holds for the motor position

$$
q_{m}=G_{d} v_{d}+G_{f} q_{m}^{d}+G_{d} d
$$

With a gradient it holds

$$
\dot{\boldsymbol{\theta}}(t)=-2 \rho G_{d}(s)\left[\boldsymbol{w}^{T} e(t)\right] .
$$

Now the filter of the adaptive algorithm $\hat{G}_{x}(t)$ becomes the disturbance transfer function $\hat{G}_{d}(s)$ of the closed loop system.

2) Adaptation with feedforward control: The PMSM has to have a good disturbance rejection but it also has to follow the reference trajectories perfectly. This can be improved, if a feedforward controller is used, which consists of the inverse model of the PMSM. The block diagram of the complete control system is shown in figure 6. It holds for the motor position with location of intervention of the adaptive controller on position level

$$
q_{m}=G_{d} d+F v_{f}+F q_{m}^{d} .
$$

For the adaptation we make the system fictively free from the reference trajectory, which is achieved with

$$
e=q_{m}-F q_{m}^{d}
$$

With assumption 2 the error for the adaptation becomes

$$
e(t)=G_{d}(s)\left[\boldsymbol{w}^{T} \boldsymbol{\theta}^{*}\right]+F(s)\left[\boldsymbol{w}^{T} \boldsymbol{\theta}(t)\right] .
$$

With a gradient it holds

$$
\dot{\boldsymbol{\theta}}(t)=-2 \rho F(s)\left[\boldsymbol{w}^{T} e(t)\right]
$$

Due to the inverse model at the input, the transfer function $\hat{G}_{x}(t)$ of figure 5 is just the filter of the feedforward controller, which is needed to differentiate the signals. For real-time applications this is an advantage, because the order of the filter is low and so computing time is saved. Another advantage is that there is no information needed of the basis controller. The disadvantage of the setup is again that the adaptation does not have constant states at accelerated movements, which makes

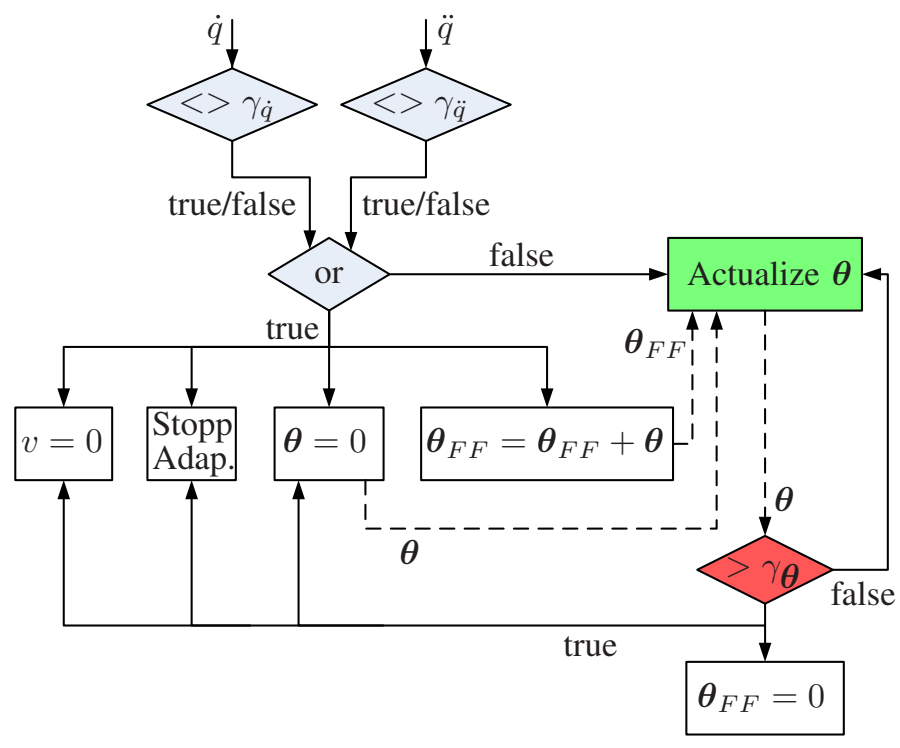

Fig. 7. Flow-chart for the logic control of the adaptation.

the convergence quite slow. If the location of intervention is chosen to be current level it holds for the motor position

$$
q_{m}=G_{d} d+G_{d} v_{d}+F q_{m}^{d} .
$$

With a gradient it holds

$$
\dot{\boldsymbol{\theta}}(t)=-2 \rho G_{d}(s)\left[\boldsymbol{w}^{T} e(t)\right]
$$

Now the filter of the adaptive algorithm $\hat{G}_{x}(t)$ becomes the disturbance transfer function $\hat{G}_{d}(s)$ of the closed loop system, as in equation (46).

\section{B. Logic control of the adaptation}

There are several reasons to stop the adaptation. The oscillations only occur at a certain velocity region, see figure 2 , so at velocity regions, where the ripples do not have any effect the adaptation can be switched off. Also at movements with high accelerations the effect of the ripples is very small. It makes sense to use the already learned information for the next learning phase. This is achieved with the following equation

$$
v(t)=\boldsymbol{\theta}(t) \boldsymbol{w}^{T}(t)+\boldsymbol{\theta}_{F F} \boldsymbol{w}^{T}(t)
$$

and with the help of figure 7 . If the adaptation is switched off the actual values of the adaptive states are set to $\boldsymbol{\theta}_{F F}$, so in the next run $\boldsymbol{\theta}$ only has to learn the oscillation which is left.

\section{Experimental results on a PMSM testbed}

1) Differences to the basis control system: Figure 8 shows in the first column experimental results of a PMSM, controlled with a common PID position controller. At constant velocity there is a clear oscillation visible. The bottom picture of the first column shows a position based Fourier analysis of this signal, which shows a peak with $N=24$.

The second column shows the compensated case, where the oscillation is canceled totally, which is also visible at the frequency spectra. The adaptive states converge in about $2 s$. 

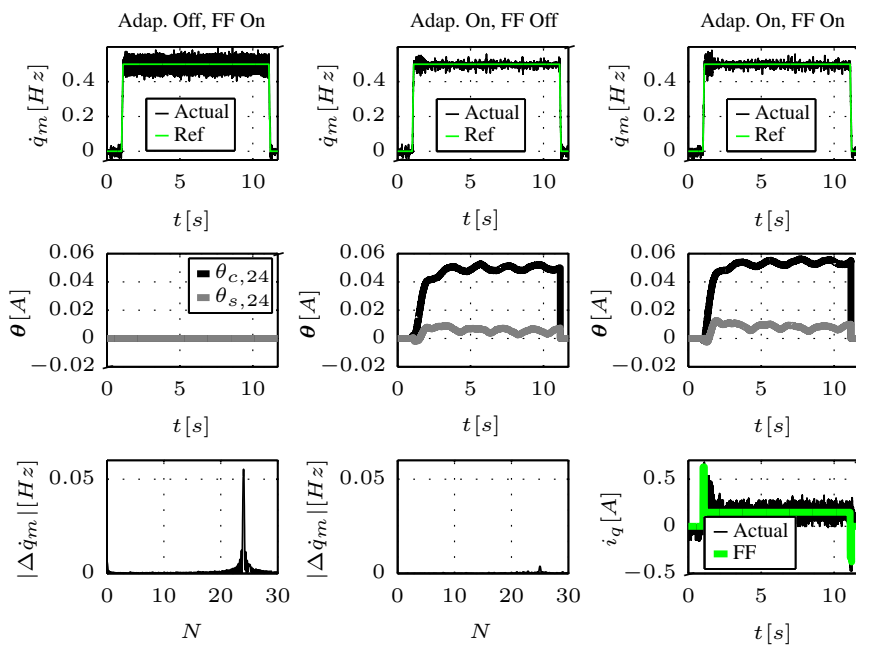

Fig. 8. Experimental results of the $\boldsymbol{G}^{-1}$ algorithm at constant speed.

The last column shows the same movement with feedforward control, which consists of the inverse model and a friction model. The offline calculated friction compensation works well, as shown in the last picture of the last column, but has no big influence on the adaptive states.

The experiments show some oscillations of the adaptive states in steady state behavior, which is probably due to a model error, non suppressed harmonics or frequency errors.

2) Convergence during an operational profile: Figure 9 (upper right) shows a practical operational profile for a PMSM. Here the logic control of the adaption from section IV-B can be studied. The second column shows that the convergence time for the $N=4$ ripple is not enough during the acceleration, but during the movement with constant speed for the adaptation it is easier to find the correct states due to the correct reset and feedforward compensation.

The first picture of the second column shows the position error during a positioning movement. Due to the feedforward controller, which includes the inverse model, this error becomes much smaller. The adaptive controller is designed to reject several harmonics as in [9].

\section{CONCLUSION}

We presented an adaptive control scheme for a PMSM, which was added to the existing control scheme and can be combined with a feedforward controller, which consists of the inverse plant. The torque ripples totally vanish with the adaptive controller, which was tested on a real time PMSM testbed.

As future work we want to implement this algorithm for more complicated systems like an elastic robot, which will have more challenges, because the system gets nonlinear and has multiple inputs and outputs.
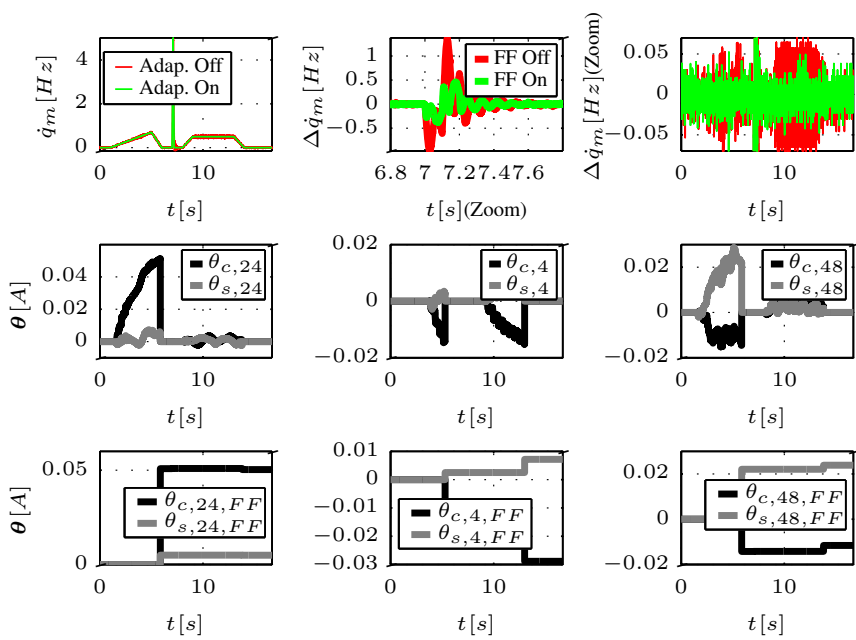

$t[s]$

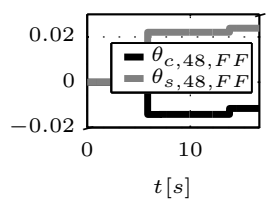

Fig. 9. Experimental results of the $G^{-1}$ algorithm during a practical operational profile, where multi harmonics are rejected.

\section{REFERENCES}

[1] M. Bodson and S. C. Douglas. Adaptive algorithms for the rejection of sinusoidal disturbances with unknown frequency. Automatica, 33:22132221, 1997.

[2] P.L. Chapman, S.D. Sudhoff, and C.A. Whitcomb. Multiple reference frame analysis of non-sinusoidal brushless dc drives. IEEE Transaction on Energy Conversion, 14(3):440-446, 1999.

[3] S.-H. Choi, J.-S. Ko, I.-D. Kim, J.-S. Park, and S.-C. Hong. Precise position control using a pmsm with a disturbance observer containing a system parameter compensator. IEE Proceedings-Electric Power Applications, 152(6):1573-1577, 4 Nov. 2005.

[4] W.-C. Gan and Li Qiu. Torque and velocity ripple elimination of ac permanent magnet motor control systems using the internal model principle. 9(2):436-447, 2004.

[5] Xiuyan Guo and M. Bodson. Adaptive cancellation of a sinusoidal disturbance with rapidly varying frequency using an augmented error algorithm. In Proc. and 2005 European Control Conference Decision and Control CDC-ECC '05. 44th IEEE Conference on, pages 48034808, 12-15 Dec. 2005.

[6] J. Holtz and L. Springob. Identification and compensation of torque ripple in high-precision permanent magnet motor drives. 43(2):309320, April 1996.

[7] T.M. Jahns and W.L. Soong. Pulsating torque minimization techniques for permanent magnet ac motor drives-a review. 43(2):321-330, 1996.

[8] H. K. Khalil. Nonlinear Systems. Prentice Hall, 2002.

[9] S. Maier and M. Bodson. Periodic disturbance rejection on a laser beam stabilizing system with adaptive controllers. In Proc. International Conference on Noise and Vibration Control (ISMA), 2008.

[10] Y.A.-R.I. Mohamed. A newly designed instantaneous-torque control of direct-drive pmsm servo actuator with improved torque estimation and control characteristics. 54(5):2864-2873, 2007.

[11] V. Petrovic, R. Ortega, A.M. Stankovic, and G. Tadmor. Design and implementation of an adaptive controller for torque ripple minimization in pm synchronous motors. 15(5):871-880, 2000.

[12] D. Schroeder. Elektrische Antriebe 2, Regelung von Antrieben. Springer Verlag, 1 edition, Januar 1995.

[13] Jian-Xin Xu, S.K. Panda, Ya-Jun Pan, Tong Heng Lee, and B.H. Lam. A modular control scheme for pmsm speed control with pulsating torque minimization. 51(3):526-536, 2004.

[14] Jong Pil Yun, ChangWoo Lee, SungHoo Choi, and Sang Woo Kim. Torque ripples minimization in pmsm using variable step-size normalized iterative learning control. In Proc. IEEE Conference on Robotics, Automation and Mechatronics, pages 1-6, 2006.

[15] Z.Q. Zhu and D. Howe. Influence of design parameters on cogging torque in permanent magnet machines. 15(4):407-412, Dec. 2000. 